\title{
Managemententwicklung - ein etwas anderes Führungsseminar in den Psychiatrischen Diensten Thurgau
}

\author{
U. Biendarra, K. Studer
}

Managementtrainings lassen sich heute von renommierten und weniger renommierten Anbietern einkaufen und Führungskräften zuteil werden. Angeboten wird im weitesten Sinne alles: vom Leadership-Seminar über Coaching, Kommunikationstraining, Strategisches Management bis zum Rhetorik-Seminar. Angesichts der Vielzahl derartiger Angebote und deren Beliebtheit sollte man annehmen, dass Führungskräfte im oberen und mittleren Kader gut gerüstet mit den Anforderungen ihres Arbeits- und Führungsalltags umgehen können. Aber genau hier - beim Transfer des Gelernten und bei der Umsetzung in die organisationsspezifische Wirklichkeit - scheitern vielfach die Anstrengungen zur Verbesserung des Führungsverhaltens. In den meisten Organisationen ist die praktizierte Führungskultur fest verankert, so dass die erlernten Fähigkeiten gar nicht zur Anwendung kommen können.

Aus diesem Grund haben die Psychiatrischen Dienste Thurgau im Zuge ihrer fortlaufenden Qualitätsbemühungen ein etwas anderes Managementseminar entwickelt und stärken damit bereits im 2. Jahr erfolgreich ihre Führungspersönlichkeiten.

\section{Die Situation}

Angesichts der sinkenden Bereitschaft der Auftraggeber und Kostenträger, die medizinischpsychiatrische Versorgung mit ungebremsten Zuwachsraten zu finanzieren, sind Verwaltungsund Spitaldirektoren heute mehr denn je zum Sparen und $\mathrm{zu}$ unkonventionellen Lösungsstrategien aufgerufen. Organisationsstrategien müssen angepasst und auf die obere und mittlere Führungsebene übertragen werden. Dies erfordert von der angesprochenen Zielgruppe ein hohes Mass an Kenntnissen zur politisch-wirtschaftlichen Situation im Kanton, betriebswirtschaftliche Kenntnisse und die Bereitschaft, sich hier gemeinsam stark zu machen.

Neben den wirtschaftlichen Veränderungen haben sich auch die Mitarbeitenden und die Patientinnen und Patienten im Verlauf der letzten Jahre deutlich verändert. Es wird zunehmend schwieriger, engagierte und motivierte Mitarbeitende zu finden, die im Zeitalter des Sparens bereit sind, Einsatz zu zeigen. Überstunden, Löhne, die im kantonalen Vergleich besser sein könnten, erhöhen nicht unbedingt die Motivation. Auch erfreut sich der Kanton Thurgau wie kaum ein anderer eines starken Bevölkerungswachs- tums. Adoleszentenkrisen, Krisen in der Lebensmitte, familiäre Konflikte, Demenzerkrankungen und Abhängigkeitserkrankungen haben wie in der ganzen Schweiz stark zugenommen.

Das Personal ist zunehmend gefordert, sich mit quantitativ mehr Patientinnen und Patienten und qualitativ anspruchsvolleren Krankheitsbildern auseinanderzusetzen. Dazu werden Führungskräfte benötigt, die die Teamentwicklung fördern und gewillt sind, Motivationsfunktion und Verantwortung zu übernehmen. Ein Managementseminar soll die angesprochene Zielgruppe in der Weiterentwicklung ihrer Kompetenzen unterstützen, damit diese ihren Arbeitsalltag autonom bewältigen kann.

\section{Das Projekt}

Im Mai 2001 wurde das Projekt «Managemententwicklung» durch die Klinikleitung der Psychiatrischen Dienste Thurgau in Auftrag gegeben. Die Ausgangslage wurde folgendermassen definiert:

- Eine Anzahl von Mitarbeitenden (etwa 10 Personen pro Jahr) wird in Kaderpositionen im ärztlich-psychotherapeutischen, pflegerischen, Hotellerie- oder Verwaltungsbereich befördert, ohne eine Führungsausbildung erhalten zu haben oder über organisatorisches/ betriebswirtschaftliches Basiswissen zu verfügen.

- Kaderpersonal, das seine Funktion schon länger innehat, erhält ausser durch spontane Rückmeldungen oder Rückmeldungen in den jährlich stattfindenden Beurteilungsund Förderungsgesprächen kein Feedback der Unterstellten zu ihrem Führungsverhalten.

- Eine gemeinsame Haltung des Kaders zu Fragen der Organisationsentwicklung ist punktuell vorhanden, wird aber nicht systematisch entwickelt und gepflegt. Dies führt dazu, dass Organisationsentwicklung und Qualitätsmanagement noch selten ein Thema der Mitarbeiterführung ist. 
Daraus wurden folgende Ziele abgeleitet:

- Es gibt Fort- und Weiterbildungsangebote, die Grundwissen in Mitarbeiterführung, Qualitätsmanagement, Betriebswirtschaftslehre und Organisationslehre vermitteln. Neben der Wissensvermittlung steht mindestens ebenbürtig die Entwicklung des persönlichen Führungsstils. Diese Angebote sind speziell auf die Psychiatrischen Dienste der Spital Thurgau AG zugeschnitten, stehen aber auch Mitarbeitenden anderer psychiatrischer Kliniken offen. Die Angebote werden auf ihre Wirksamkeit überprüft.

- Es gibt Instrumente zur Potentialbeurteilung von Mitarbeitenden, die für Kaderpositionen oder Beförderungen vorgesehen sind.

- Es gibt ein System zur Beurteilung von Führungsverhalten.

Im Rahmen eines Echoraums im Juli 2001 wurde einigen Mitarbeitenden aller Hierarchiestufen die Gelegenheit gegeben, sich über das geplante Programm genauer zu informieren, Erfahrungen auszutauschen sowie Wünsche, Vorstellungen und Bedenken zu äussern. Insgesamt beurteilten die 21 Geladenen ein derartiges Seminar als wünschenswerte Weiterentwicklung im Bereich Führung.

Nach Erarbeitung eines Grobkonzepts mit einem externen Organisationsberater stellten die Projektleiterinnen eine zwölfköpfige Projektgruppe zusammen, die sie aus Bewerbungen zu diesem Projekt akquirierten. Bei der Auswahl der Teilnehmenden wurde Wert darauf gelegt, möglichst alle Berufsgruppen und alle Hierarchiestufen in der Gruppe zu vereinen.

In einem ersten Schritt innerhalb der Projektarbeit wurde eine Ist-Analyse mit den folgenden Kernfragen erarbeitet: Was ist gut/nicht gut an der bestehenden Führungskultur? Wie werden Führungskräfte ausgewählt? Wie werden Entscheidungen vorbereitet und schliesslich gefällt? Wie laufen Informationen und Kommunikation?

Die Projektgruppe konstatierte einhellig folgende organisatorische Problembereiche mit hohem Verbesserungsbedarf:

- gemeinsame strategische Grundhaltung/koordinierte therapeutische Angebotsplanung;

- bereichsinterne und -übergreifende Zusammenarbeit und Unterstützung;

- mehr Verständnis für die Belastung im Arbeitsalltag;

- transparente Entscheidungswege statt 4Augen-Gespräche;

- Klärung von Verantwortungs- und Kompetenzbereichen für einzelne Funktionen;
- von der sternförmigen zur zirkulären Kommunikation und Entscheidung;

- mehr betriebswirtschaftliches Verständnis und Erweiterung der Verantwortung und des Handlungsspielraums, z.B. bei der Genehmigung von Fort- und Weiterbildungen, Mitspracherecht bei Einstellungen.

Besuche in anderen Kliniken und KMUs, Impulsreferate und Diskussionen mit externen Referenten verdeutlichten, dass diese Veränderungen nur durch die parallele Förderung individueller Fähigkeiten und Prüfung der bestehenden Kultur und der organisationsspezifischen Bedingungen zu realisieren seien. Zur Gestaltung dieser parallelen Ausrichtung des Programms teilte sich die Projektgruppe in vier Gruppen und erarbeitete mit der Klinikleitung Teilkonzepte zu Form, Inhalt, Umwelt und den Rahmenbedingungen eines geeigneten Programms.

Im März 2002 konnte die Projektgruppe der Klinikleitung ein ausgearbeitetes Konzept eines Managemententwicklungsprogramms vorlegen, das mit den nötigen Anträgen versehen war. Als treibende Kraft dieses Programms genehmigte diese alle Anträge. Das Managemententwicklungsprogramm, zukünftig nur noch MEP genannt, sollte in einem ersten Durchlauf erprobt werden.

\section{MEP 1: Organisation und Durchführung}

Als Auftraggeber definierte die Klinikleitung die Zielgruppe im ersten Durchgang: die leitenden Ärzte/Ärztinnen der Klinik, der Externen Psychiatrischen Dienste, des Kinder- und Jugendpsychiatrischen Dienstes, die Bereichsleitungen der Pflege sowie der Hotellerie und Verwaltung, insgesamt dreizehn Personen. Diese wurden im Rahmen einer offiziellen Veranstaltung über das Programm informiert.

Die organisatorischen Rahmenbedingungen wurden gesteckt. Dazu gehörte die Akquirierung einer externen Leitung, eines im Profit- und Non-Profit-Unternehmen langjährig erfahrenen Organisationsberaters, der bereits im Vorfeld an der Erarbeitung des Grobkonzepts mitgearbeitet hatte, sowie einer internen Leitung, die sich für diese Aufgabe beworben hatte und als Psychologin im Stab Unternehmensentwicklung arbeitete. Durch die Kombination einer externen und internen Leitung sollte fachliche Kompetenz und interne Kommunikation und Wissen sichergestellt werden.

Das Konzept sah vor, insgesamt sieben Workshops, verteilt über ein Jahr, in externen Semi- 


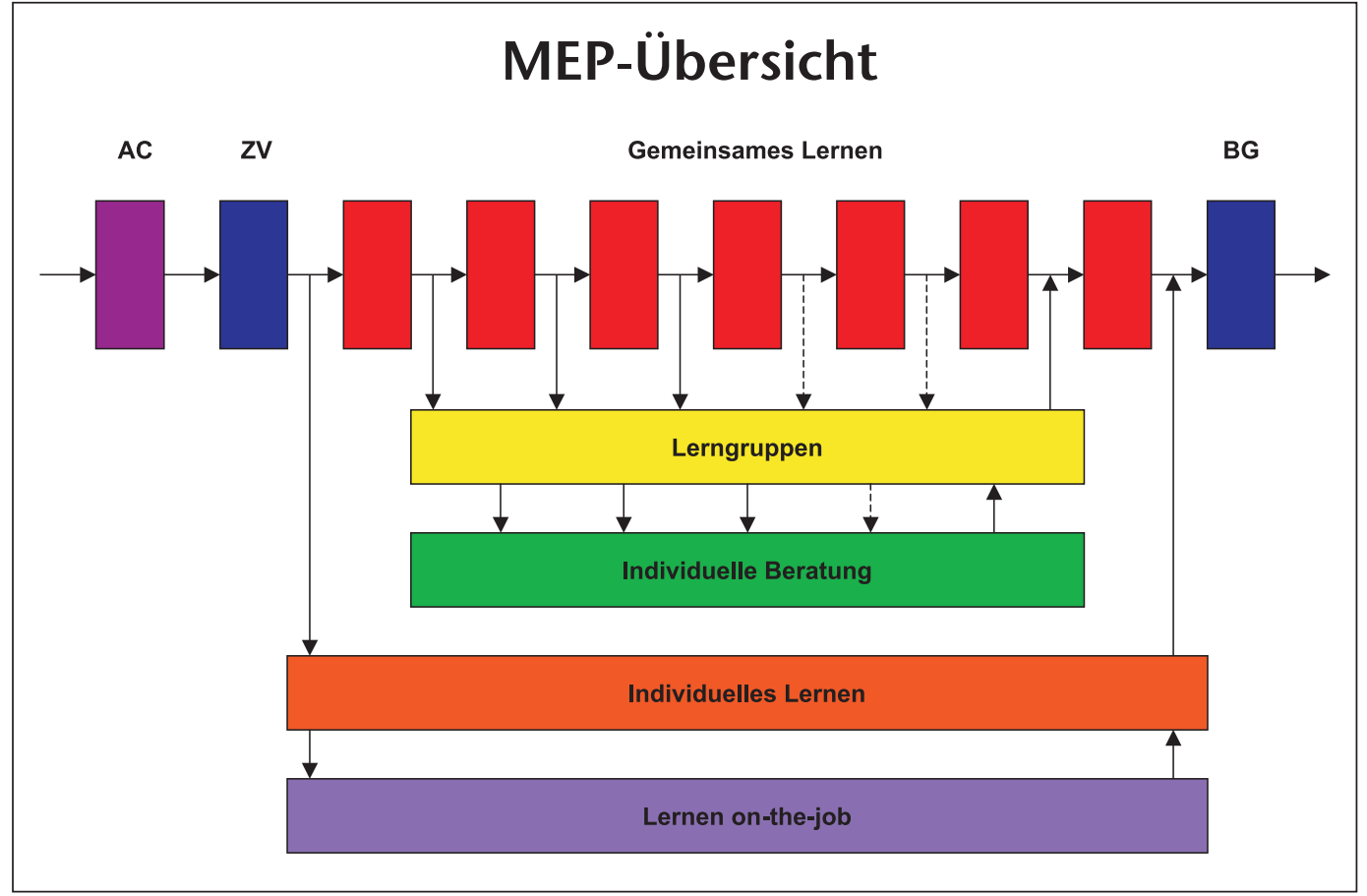

narhotels abzuhalten. Begleitend dazu sollten Lerngruppen und individuelle Beratungsgespräche mit den Leitungen des Programms stattfinden, die als Unterstützung in aktuellen und konkreten Problemen im Führungsalltag definiert wurden. Zusätzlich sollten jedem/jeder Teilnehmer/in vier Stunden pro Woche für das Lernen am Arbeitsplatz zur Verfügung gestellt werden, in denen er/sie mit Unterstützung des/ der direkten Vorgesetzten Gelerntes erproben und umsetzen konnte.

Im Januar 2003 wurde das MEP mit individuellen Assessments, die jeder/jede der dreizehn Teilnehmer/innen zu absolvieren hatte, gestartet. Diese sollten Informationen zu den Dimensionen Persönlichkeits-, Sozial- und Führungskompetenz liefern und wurden nicht zu Selektionszwecken eingesetzt. Ein externer Anbieter führte mit den Teilnehmenden gezielt zusammengestellte Übungen und Aufgaben durch, deren Ergebnisse in die folgenden Lernzielvereinbarungsgespräche einflossen. Hier wurden die individuell zu erreichenden Lernziele mit externer und interner Leitung, dem/der direkten Vorgesetzten des/der betreffenden Teilnehmers/-in und dem/der Teilnehmer/in im Sinne eines Lernvertrags vereinbart. Lernziele wie die Verbesserung des betriebswirtschaftlichen Know-hows, effektives Zeitmanagement, Präsentationstechniken wie Rhetoriktraining, -techniken, Selbstmarke- ting, Entwicklung einer funktionierenden bereichsinternen und -übergreifenden Zusammenarbeit, Verbesserung der Kommunikation durch gezielte Anleitung in schwierigen Gesprächen, Persönlichkeitsentwicklung und Aspekte von Leadership wurden identifiziert, geclustert und als Grundlage für die Gestaltung der Workshops aufgearbeitet.

Vor dem Hintergrund dieser Informationen wurden nun die Ziele der MEP-Teilnehmenden noch einmal konkretisiert und definiert. Im Verlauf des MEP haben die Teilnehmenden:

- die Rückmeldungen aus den Assessments über ihre Stärken und Schwächen in den genannten Dimensionen verarbeitet;

- an ihren vereinbarten Lernzielen gearbeitet und Impulse für Verhaltensmodifikationen im Alltag erprobt, reflektiert, umgesetzt und damit ihre Wirkung in den Psychiatrischen Diensten und deren Unternehmenskultur verbessert;

- an Jahresthemen der Direktion gearbeitet und damit einen Beitrag am Erfolg der Psychiatrischen Dienste Thurgau geleistet;

- die Feedbacks der Mitarbeitenden, Kollegen/-innen, Vorgesetzten und der Leitung in bezug auf ihre Persönlichkeits-, Sozial- und Führungskompetenz erhalten und für ihre weitere Entwicklung Massnahmen vereinbart. 
Die definitiven Themen und deren Verteilung auf die nachfolgenden Workshops wurden Anfang April 2003 im ersten Workshop mit den Teilnehmenden gemeinsam erarbeitet und vereinbart. Sie basierten auf den individuellen Lernzielen und den Jahresthemen der Direktion, die als Auftrag an die Teilnehmenden in das Programm einflossen. Die Inhalte sollten sich an den strategischen Erfolgspositionen der Spital Thurgau AG und den Führungsinstrumenten der Psychiatrischen Dienste (Leitbild, Personal- und Qualitätspolitik, strategische Ziele) orientieren. Die Methoden sollten die Reflexion, die Einsicht und die Handlungsorientierung der Teilnehmenden, aber auch deren Spass am Lernen fördern. Lernen sei geprägt durch persönliches Engagement, Partnerschaftlichkeit, Eigenverantwortung und Selbststeuerung und dem Grundsatz verpflichtet: Soviel Theorie wie nötig, soviel Praxis wie möglich!

Innerhalb des Jahres 2003/2004 arbeiteten die dreizehn Teilnehmerinnen und Teilnehmer an ihren individuellen und den organisatorischen Lernzielen. Die vereinbarten Themen seien nachfolgend stichwortartig aufgelistet.

Workshop 1: Herstellung von Kontakt, Arbeitsfähigkeit und Zusammengehörigkeitsgefühl. Planung der thematischen Abfolge der Workshops und Vereinbarung der gemeinsamen Vorbereitung der Workshops. Für jeden Workshop wurden Freiwillige und/oder speziell an diesem Thema Interessierte gewählt, die gemeinsam mit den Leitungen des MEPs das Workshopprogramm vorbereiten sollten, um die Orientierung an den Lernzielen und den Praxisbezug sicherzustellen.

Workshop 2: Betriebswirtschaft: Grundlagen des Wirtschaftssystems, Bilanz und Erfolgsrechnung der Psychiatrischen Dienste Thurgau, Geschäftsbericht, Elemente der Kernprozesse wie Fort- und Weiterbildungsbudget, Investitionsbudget, Bauplanung, Kalkulationen.

Workshop 3: individuelles Zeitmanagement: Erstellung einer individuellen Arbeits- und Zeitanalyse, SWOT-Analyse des Selbstmanagements, Aktionsplan für die Zukunft.

Workshop 4: laterale und vertikale Zusammenarbeit: Vision einer Zusammenarbeit, Massnahmen, gegenseitige Kommunikation von Erwartungen, Umgang mit krisenhaften Situationen.

Workshop 5: Positionierung als Führungsrolle und Trennung von der therapeutischen Rolle, Reflexion der eigenen Rolle, Rollentraining, Positionierung in der Reihe des oberen Kaders, Durchsetzungsvermögen und -potential erkennen und umsetzen.
Workshop 6: Kommunikation: Rhetoriktraining, Kommunikation und EFQM, Anleitung in herausfordernden Gesprächen.

Workshop 7: Politik und Strategie: Psychiatrieplanung im Thurgau und Möglichkeiten der Vernetzung und Zusammenarbeit in der psychiatrischen Versorgung.

Zur Verdeutlichung der organisatorischen und der persönlichen Wirkung des MEPs sei der Workshop 5 «Positionierung» im Dezember 2003 nachfolgend exemplarisch beschrieben. Wie alle Workshops war auch dieser strukturell und organisatorisch nach dem gleichen Prinzip aufgebaut. Die zu erreichenden Ziele wurden im Vorfeld folgendermassen definiert:

Die Teilnehmenden haben

- ihre eigene Rolle als Führungskraft reflektiert, modifiziert und positioniert;

- ihr Durchsetzungsvermögen entdeckt, trainiert und angereichert;

- im Gespräch mit dem Vorsitzenden der Geschäftsleitung der Spital Thurgau AG und der Klinikleitung Wirkung erzielt.

Den Einstieg stellte ein Impulsreferat einer geladenen Referentin dar, die die Beziehung zwischen Geschlecht und Rollen, Rollenzuschreibungen und Unterschiede zwischen männlichen und weiblichen Führungsrolleninhabern thematisierte. Dieser breite Einstieg in die Thematik aktivierte eine erste Auseinandersetzung mit der eigenen Situation im Führungsalltag und ermöglichte in einem zweiten Schritt eine Vertiefung im Kleingruppengespräch mit den anderen Kolleginnen und Kollegen. «Welche Rollen habe ich? Wie lebe ich sie? Wie nehmen sie andere wahr?»

Am Nachmittag spielten die Teilnehmenden Aspekte ihrer Rolle im Rollentraining durch. Angeleitet von professionellen, sozialpädagogisch geschulten Schauspielerinnen und Schauspielern eines Unternehmenstheaters wurden konkrete Situationen aus dem Arbeits- und Führungsalltag durchgespielt. Es gelang, konkrete Szenen aus dem Führungsalltag aufzugreifen, die ein hohes Mass an Sozialkompetenz erfordern, beispielsweise die Führung schwieriger Gespräche mit Mitarbeitenden, Durchsetzungsvermögen zu signalisieren und eigene Entscheidungen vor höheren Vorgesetzten zu vertreten. Die vierstündige Sequenz war für alle Teilnehmenden anstrengend und intensiv, beinhaltete jedoch für das eigene Führungsverhalten und Durchsetzungsvermögen einen hohen individuellen Erkenntnisgewinn. Jede gezeigte Verhaltensweise wurde von der Leitung des Unternehmenstheaters kommentiert und mit jedem/jeder Teilneh- 
mer/in auf mögliche Handlungsalternativen hin geprüft. Auch der Spass am Lernen kam dabei nicht zu kurz.

Der erste Tag wurde mit einem zuvor vorbereiteten Gespräch mit dem Vorsitzenden der Geschäftsleitung der Spital Thurgau AG und der Klinikleitung beendet. Mut zum Diskurs im Umgang mit höheren Führungskräften und Verantwortungsträgern beweisen, kritische Fragen zur aktuellen Politik und Strategie im Spitalverbund stellen und Wirkung durch die eigene Meinung erzielen, stellten weitere Herausforderungen im dichten Tagesprogramm dar.

Was schlussendlich in diesem Gespräch erreicht wurde, war Gegenstand der Nachbesprechung mit der Klinikleitung am zweiten Tag des Workshops. Mit deren Unterstützung konnten einige Massnahmen vereinbart und geplant werden, die für die Zukunft der Psychiatrischen Dienste wesentlich sein werden, so z. B. die Beibehaltung der bestehenden therapeutischen Vielseitigkeit. Hier wurden Aufgaben und Verantwortliche definiert, die diese Entwicklung zukünftig weiter vorantreiben werden. Auch die Mitgliedschaft mindestens eines/einer ärztlichen Vertreters/-in in der Geschäftsleitung der Spital Thurgau AG wurde als essentiell für die Interessenvertretung und Weiterentwicklung der Psychiatrischen Dienste hervorgehoben und darf nicht ausschliesslich wirtschaftlichen Gedanken geopfert werden.

Der weitere Verlauf des Tages wurde in Kleingruppenarbeiten gestaltet. Die Teilnehmenden wurden aufgefordert, ihre Rolle und Funktion in der MEP-Gruppe nach mittlerweile fünf Workshops einzuschätzen und sich auf einer quer durch den Raum verlaufenden Linie auf gedachten Positionen 1-13 zu positionieren. Das dabei notwendige Durchsetzungsvermögen bei der Verteidigung der eigenen Position überraschte einzelne Teilnehmende im positiven wie auch im negativen Sinn. Die Ergebnisse wurden dahingehend aufgearbeitet, für die Zukunft Verhaltensmodifikationen für ein besseres Durchsetzungsvermögen oder für mehr Zurückhaltung in spezifischen Situationen zu planen und diese im Arbeitsalltag zu erproben.

Am späten Nachmittag wurde der Workshop mit einer Fotolanguage beendet, die die Funktion einer Feedbackrunde hatte.

Im April 2004 war das MEP 1 mit Bilanzgesprächen beendet. Diese wurden im gleichen Setting wie die Lernzielvereinbarungsgespräche durchgeführt, d.h. mit externer und interner Leitung, dem/der direkten Vorgesetzten und dem/der Teilnehmer/in selbst. Neben der Frage nach der Zielerreichung wurden auch weitere notwendige Entwicklungsschritte und Massnahmen thematisiert.

\section{Wirkungen}

Was die Auftraggeber derartiger umfangreicher «Weiterbildungen» immer beschäftigt, ist die Frage «Was wurde erreicht?» Dies zeichnet sich heute, nach kaum drei Monaten, bereits deutlich ab. Das obere Kader ist zu einer Gruppe zusammengewachsen, in der unterschiedliche Meinungen und Interessen im Diskurs zum Zug kommen. Strategische Fragen und Überlegungen werden in einer neu konstituierten Sitzung, die einmal im Monat stattfindet, gemeinsam angegangen. Eine Pendenzenliste wurde von den Teilnehmenden angefertigt, die nun im Verlauf der gestärkten Zusammenarbeit systematisch «abgearbeitet» wird. Auszugsweise seien hier einige Punkte genannt:

- Personallisten werden aktualisiert und dem oberen Kader zur Verfügung gestellt.

- Neue Entscheidungsmodi: zirkulär statt sternförmig.

- Delegation von Kompetenzen und Verantwortung für:

- Fort- und Weiterbildungsbudget,

- Sachbudget,

- Stellenbudget und -besetzungen,

- Leistungsprämien,

- Beförderungen.

- Erarbeitung einer neuen Sitzungskultur: weniger, komprimierter, mit Traktandenliste, ohne Störungen.

Auch das Feedback von Unterstellten ist neu und wird von den MEP-1-Teilnehmenden sogar eingefordert. Diese konstatieren eine positive Veränderung ihrer Vorgesetzten: Bessere Kooperation, mehr gegenseitige Unterstützung, mehr «good will» in der täglichen Arbeit. Zurückzuführen ist dieser Erfolg auf die Berücksichtigung einer gemeinsamen organisationsspezifischen Kultur und Wirklichkeit. Impuls- und Entwicklungsarbeit wurde in den Workshops geleistet, in den Lerngruppen und den individuellen Beratungsgesprächen wurde an konkreten Situationen aus dem Arbeitsalltag gearbeitet. Am Arbeitsplatz wurden die Teilnehmenden von ihren Vorgesetzten unterstützt. Die Klinikleitung hat dieses Programm auf den Weg gebracht, fortlaufend begleitet und überwacht. Alle Elemente dieses Programms haben sich an den vorgefundenen organisationsspezifischen Bedingungen orientiert. Die erworbenen Kompetenzen haben sich aus den Stärken und Schwächen 
der Teilnehmenden, die innerhalb der Organisation beobachtbar waren, entwickelt und fliessen wieder in die Organisation zurück.

Dank der fortlaufenden Kommunikation und Auseinandersetzung aller Beteiligten ist das Ergebnis als sehr positiv zu bewerten.

\section{MEP 2}

Und aus diesem Grund geht es auch bereits seit Januar 2004 weiter. Um die Kultur nachhaltig weiterentwickeln zu können, wurde von der Klinikleitung ein MEP 2 in Auftrag gegeben. Hier engagieren sich 35 Angehörige der nächsten Führungsebene, alle Pflegeleitungen und Therapeutischen Leiterinnen und Leiter der Stationen, der Externen Psychiatrischen Dienste, des Kinder- und Jugendpsychiatrischen Dienstes sowie Führungspersonen aus Hotellerie und Verwaltung mit ihren spezifischen Problemen und Anforderungen, die sich auf dieser Hierarchiestufe ergeben. Das Konzept wurde bis auf die Anzahl der Workshops nicht verändert. Den jetzigen Teilnehmenden stehen fünf Workshops zur Weiterentwicklung zur Verfügung. Diese werden in externen Seminarhotels abgehalten. Auch sie arbeiten in Lerngruppen an konkreten Situationen aus dem Arbeitsalltag und führen individuelle Beratungsgespräche mit der Leitung des Programms, die für diesen Zyklus erneut engagiert werden konnte. Gleichzeitig setzen sie am Arbeitsplatz mit Unterstützung ihrer Vorgesetzten, dies sind nun die MEP-1-Teilnehmenden, das Gelernte um. Start und Ende des MEP 2 stellen wiederum die individuellen Lernzielvereinbarungs- und Bilanzgespräche dar. Angesichts der grossen Teilnehmerzahl wurde für diesen Durchgang auf die Durchführung von Einzelassessments verzichtet. Grundlage der indivi- duellen Lernziele sind die jährlich stattfindenden Beurteilungs- und Förderungsgespräche, die durch Zusatzkriterien zur Beurteilung der Persönlichkeits-, Sozial- und Führungskompetenz ergänzt wurden. Auch in dieser Gruppe mangelt es nicht an der nötigen Bereitschaft und Motivation, zumal auch hier das Engagement der Klinikleitung deutlich spürbar ist. In regelmässigen Abständen nehmen einzelne Personen der Klinikleitung an Teilen der Workshops teil. Und auch die MEP-1-Teilnehmenden bereichern das Programm mit ihrem Input. Dies soll die vertikale Zusammenarbeit und die Kommunikation auf Dauer stärken und verbessern sowie die in die Wege geleiteten Veränderungen zur Umsetzung bringen. So verstanden steht neben der Vermittlung individueller Fähigkeiten und Kompetenzen eine Weiterentwicklung der Psychiatrischen Dienste im Vordergrund. Autonome und selbständige Mitarbeitende, die ihren Beitrag am Erfolg der Psychiatrischen Dienste leisten, sind das Ziel.

\section{Ausblick}

Im Jahr 2005 beginnt die Klinik mit einem dritten Durchlauf, der für Angehörige externer Institutionen geöffnet werden soll. Im Sinne eines Grundgedankens des QM, von den Besten $\mathrm{zu}$ lernen und Wissen auch selber weiterzugeben, wollen die Psychiatrischen Dienste ihre Erfahrungen und ihr erworbenes Know-how gerne mit anderen Kliniken teilen.

\section{Interessenten/Bewerbungen MEP 3:}

Dr. K. Studer

Psychiatrische Dienste Thurgau

Postfach 154

8596 Münsterlingen 